14.3

\title{
Моделирование кинетических кривых полимеразной цепной реакции, полученных с помощью флуоресцентных олигонуклеотидных зондов*
}

\author{
(C) А.А. Фёдоров, ${ }^{1}$ Д.Г. Сочивко, ${ }^{2}$ Д.А. Варламов, ${ }^{3}$ В.Е. Курочкин ${ }^{1}$ \\ ${ }^{1}$ Институт аналитического приборостроения РАН, \\ 190103 Санкт-Петербург, Россия \\ 2 ООО „Синтол“, \\ 127550 Москва, Россия \\ ${ }^{3}$ Всероссийский научно-исследовательский институт сельскохозяйственной биотехнологии, \\ 127550 Москва, Россия \\ e-mail: f_aa@mail.ru
}

(Поступило в Редакцию 11 января 2018 г.)

В настоящее время имеется множество моделей полимеразной цепной реакции, в том числе претендующих на точные количественные оценки результатов реакции. При этом в подавляющем большинстве моделей кинетика накопления продукта реакции и кинетика сигнала флуоресцентного репортера считаются тождественными. Предложена модель полимеразной цепной реакции, позволяющая исследовать различие данных функций в случае использования гибридизационного зонда как источника регистрируемого флуоресцентного сигнала. Проверена адекватность модели, продемонстрировано, что кинетика накопления продукта и зондового репортера могут значительно различаться.

DOI: 10.21883/JTF.2018.10.46489.6-18

\section{Введение}

Метод полимеразной цепной реакции (ПЦР), известный уже более тридцати лет, является на сегодняшний день одним из основных аналитических инструментов в генетических исследованиях. В ходе ПЦР при участии фермента происходит циклическое копирование целевого фрагмента молекулы ДНК. С помощью метода полимеразной цепной реакции в реальном времени (ПЦР-РВ) проводят количественный анализ специфических фрагментов ДНК. В ПЦР-РВ синтез новых цепей ДНК сопровождается ростом флуоресцентного сигнала от репортера того или иного типа, что позволяет оптически зарегистрировать ход накопления продукта синтеза. Получаемые в координатах 〈флуоресценция〉〈количество циклов〉 кривые приобретают характерную S-образную форму, поскольку реакция синтеза новых копий ДНК останавливается вследствие исчерпания ресурсов реакционной смеси. По некоторым интегральным характеристикам этой кривой оказывается возможным оценить исходную концентрацию ДНК, если параллельно получены подобные кривые для известных концентраций данной ДНК. На этом строится современная программно-алгоритмическая обработка и анализ данных ПЦР-РВ анализа.

Если на качественном и практическом уровнях механизм реакции и вклад основных составляющих в результат ПЦР изучен достаточно хорошо, то полноценного количественного описания процесса ПЦР, позволяющего по заданным параметрам реакции смодели-

\footnotetext{
* Вторая российская конференция с международным участием „Физика - наукам о жизни“ (Санкт-Петербург, 18-22 сентября 2017 г.)
}

ровать кинетическую кривую сигнала флуоресцентного репортера, на данный момент нами не обнаружено. Появление подобного математического аппарата позволило бы создать хороший научно-технический задел для дальнейшего развития методов обработки и анализа результатов ПЦР.

В настоящее время предложено множество различных моделей ПЦР. Модели количественного описания процесса синтеза ДНК в ПЦР основываются на дифференциальных уравнениях химической и ферментативной кинетики [1-4]. В рамках данного подхода был предложен целый ряд моделей, исследующих влияние различных факторов на ПЦР [1-4]. Практически во всех работах явно или не явно считалось, что экспериментальные и модельные кинетические кривые ПЦР, первые из которых являются кривыми накопления сигнала флуоресценции, а вторые - кривыми накопления копий исходного фрагмента ДНК, можно считать тождественными. Однако такое отождествление представляется нам не совсем правильным. Как было отмечено выше, для приборной визуализации накопления продуктов ПЦР используются флуоресцентные репортеры, т. е. экспериментальные кривые ПЦР-РВ фактически отражают не накопление продукта реакции, а кинетику флуоресцентного сигнала используемых репортеров. На практике используются два способа флуоресцентной регистрации результатов ПЦР.

Первый способ - использование специфических к анализируемой ДНК-мишени одноцепочных олигонуклеотидных последовательностей, несущих флуорофор и тушитель флуоресценции. Данные конструкции называются гибридизационными зондами. В процессе синтеза ферментом комплементарной цепи ДНК гибридизован- 
ный зонд расщепляется за счет экзонуклеазной активности ДНК-полимеразы, тушитель и гаситель пространственно разделяются, что приводит к формированию флуоресцентного отклика системы.

Вторым способом визуализации результатов ПЦР-РВ является использование интеркалирующих красителей (ИК) - соединений, способных к значительному усилению флуоресцентного сигнала в результате обратимого связывания с двухцепочными фрагментами ДНК. Однако есть как минимум две причины, по которым кинетика накопления сигнала ИК и синтеза цепей не совпадают. Во-первых, ИК могут выявлять различные неспецифические ПЦР продукты и реакции. Во-вторых, некоторые широко используемые на практике красители могут как ингибировать ПЦР, так и „выгорать“ в течение процесса ПЦР, внося искажения в информационный сигнал [5].

В связи с указанными причинами считается, что использование зондов позволяет получить кинетическую кривую, более близкую к кинетике накопления продуктов ПЦР. Повторимся, что в работах по моделированию ПЦР разница между экспериментальными кривыми и кривыми накопления ДНК не учитывается, хотя для получения экспериментальных кривых используются как ИК - в основном SYBR Green [6-9], так и зонды [3]. В лучшем случае авторы используют простую линейную зависимость для взаимного пересчета этих величин $[8,9]$.

Задача настоящей работы - предложить математическую модель ПЦР-РВ, которая позволит сравнить кинетические кривые накопления самого продукта ПЦР и кривые, полученные с помощью „визуализирующих“ продукт ПЦР флуоресцентных зондов.

\section{Модель формирования флуоресцентного сигнала зонда}

Рассматриваются следующие основные компоненты и стадии процесса ПЦР, происходящие в течение одного цикла ПЦР. На первой стадии цикла - нагревание реакционной смеси выше $90^{\circ} \mathrm{C}$ - происходит денатурация двухцепочечной ДНК на две комплементарные одноцепочечные молекулы $-F$ и $F^{\prime}$. Далее смесь охлаждается, наступает стадия отжига, на которой специфические к $5^{\prime}$-концевому участку каждой из цепей праймеры $\operatorname{Pr}$ и $\operatorname{Pr}^{\prime}$ имеют возможность сесть на цепи $F$ и $F^{\prime}$. То же самое справедливо и для сигнальных зондов-репортеров $Z$ и $Z^{\prime}$, которые также садятся на свои участки цепей $F$ и $F^{\prime}$. Посадка зондов и праймеров являются независимыми событиями. Фермент ДНК-полимераза связывается с комплексом праймера и цепи. Далее наступает черед стадии элонгации, на которой фермент, двигаясь от праймера в сторону участка посадки зонда и далее до конца цепи, начинает достраивать новую комплементарную цепь. В случае успешного завершения синтеза одноцепочная молекула ДНК $\left(F\right.$ или $\left.F^{\prime}\right)$ превращается в двухцепочечную молекулу - копию исходной $\left(F \| F^{\prime}\right)$.

Если в процессе синтеза зонд гибридизован с одноцепочечным фрагментом, то он расщепляется и его флуо- ресцентная метка становится активной. Флуоресцентный сигнал от всех активных меток на данном цикле даст точку на кинетической кривой, строящейся в координатах 〈флуоресценция〉-〈номер цикла $\rangle$. Если же зонд не сядет на положенное место, то синтез может пройти и без него, тогда новый двухцепочечный фрагмент ДНК не будет давать вклад во флуоресцентный сигнал реакции. Также две исходные цепи $F$ и $F^{\prime}$ могут соединиться обратно в исходную двойную цепь, даже если на одну из них или на обе успели сесть праймеры или зонды, но элонгация еще не началась. Данная реакция принимается за необратимую, поскольку при типичной температуре элонгации диссоциация таких комплексов практически не происходит. Введем следующие обозначения промежуточных комплексов данной реакции:

$S$ и $S^{\prime}$ - комплексы фрагмента ДНК и соответствующего праймера $\left(S=F+\operatorname{Pr}, S^{\prime}=F^{\prime}+\operatorname{Pr}^{\prime}\right)$;

$F Z$ и $F Z^{\prime}$ - комплексы фрагмента ДНК и соответствующего зонда. Здесь и далее не будем использовать двойное штрихование и будем писать $F Z^{\prime}$ вместо $F^{\prime} Z^{\prime}$;

$S Z$ и $S Z^{\prime}$ - комплексы фрагмента ДНК с соответствующими праймерами и зондами.

Bce промежуточные комплексы в случае неучастия в синтезе комплементарной цепи диссоциируют при нагревании на исходные компоненты. Для продуктов реакции введем такие обозначения:

$P_{1}$ и $P_{1}^{\prime}-$ продукт - двойная цепь, полученная синтезом и не внесшая вклад во флуоресцентный отклик системы;

$P_{1} Z$ и $P_{1} Z^{\prime}-$ двойная цепь, полученная синтезом и внесшая вклад во флуоресцентный отклик системы;

$P_{2}$ - двойная цепь, полученная гибридизацией одиночных.

С точки зрения результата ПЦР - все перечисленные продукты не различаются между собой, поскольку являются просто двойными цепями $F \| F^{\prime}$. Однако вклад в информационный сигнал дадут лишь те синтезированные цепи, при синтезе которых участвовал зонд. Цепи же $P_{2}$, образовавшиеся путем гибридизации одиночных фрагментов, не дают вклада ни в сигнал, ни в прибавку новых цепей на цикле реакции, а только вхолостую снижают количество доступного субстрата для синтеза.

С помощью принятых обозначений схему реакций описанного процесса можно представить так:

Первичные реакции

$$
\begin{aligned}
& F+\operatorname{Pr} \underset{k_{-1}}{\stackrel{k_{1}}{\longrightarrow}} S, F^{\prime}+\operatorname{Pr}^{\prime} \underset{k_{-1}}{\stackrel{k_{1}}{\longrightarrow}} S^{\prime}, \\
& F+Z \underset{k_{-1}}{\stackrel{k_{1}}{\longrightarrow}} F Z, \quad F^{\prime}+Z^{\prime} \underset{k_{-1}}{\stackrel{k_{1}}{\longrightarrow}} F Z^{\prime}, \\
& S+Z \underset{k_{-1}}{\stackrel{k_{1}}{\longrightarrow}} S Z, S^{\prime}+Z^{\prime} \underset{k_{-1}}{\stackrel{k_{1}}{\longrightarrow}} S Z^{\prime}, \\
& F Z+\operatorname{Pr} \underset{k_{-1}}{\stackrel{k_{1}}{\longrightarrow}} S Z, F Z^{\prime}+\operatorname{Pr}^{\prime} \underset{k_{-1}}{\stackrel{k_{1}}{\longrightarrow}} S Z^{\prime}, \\
& F+F^{\prime} \stackrel{k_{2}}{\longrightarrow} P_{2}, F+S^{\prime} \stackrel{k_{2}}{\longrightarrow} P_{2}+\operatorname{Pr}^{\prime}
\end{aligned}
$$




$$
\begin{gathered}
F^{\prime}+S \stackrel{k_{2}}{\longrightarrow} P_{2}+\operatorname{Pr}, F+F Z^{\prime} \stackrel{k_{2}}{\longrightarrow} P_{2}+Z^{\prime}, \\
F^{\prime}+F Z \stackrel{k_{2}}{\longrightarrow} P_{2}+Z, F+S Z^{\prime} \stackrel{k_{2}}{\longrightarrow} P_{2}+\operatorname{Pr}^{\prime}+Z^{\prime}, \\
F^{\prime}+S Z \stackrel{k_{2}}{\longrightarrow} P_{2}+\operatorname{Pr}+Z .
\end{gathered}
$$

Вторичные реакции

$$
\begin{gathered}
S+S^{\prime} \stackrel{k_{2}}{\longrightarrow} P_{2}+\operatorname{Pr}^{\prime}+\operatorname{Pr}, S+F Z^{\prime} \stackrel{k_{2}}{\longrightarrow} P_{2}+\operatorname{Pr}+Z^{\prime}, \\
S^{\prime}+F Z \stackrel{k_{2}}{\longrightarrow} P_{2}+\operatorname{Pr}^{\prime}+Z, \\
S+S Z^{\prime} \stackrel{k_{2}}{\longrightarrow} P_{2}+\operatorname{Pr}+\operatorname{Pr}^{\prime}+Z^{\prime}, \\
S^{\prime}+S Z \stackrel{k_{2}}{\longrightarrow} P_{2}+\operatorname{Pr}+\operatorname{Pr}^{\prime}+Z, \\
F Z+F Z^{\prime} \stackrel{k_{2}}{\longrightarrow} P_{2}+Z+Z^{\prime}, \\
F Z+S Z^{\prime} \stackrel{k_{2}}{\longrightarrow} P_{2}+Z+\operatorname{Pr}^{\prime}+Z,^{\prime} \\
F Z^{\prime}+S Z \stackrel{k_{2}}{\longrightarrow} P_{2}+Z+\operatorname{Pr}+Z^{\prime}, \\
S Z+S Z^{\prime} \stackrel{k_{2}}{\longrightarrow} P_{2}+Z+\operatorname{Pr}+\operatorname{Pr}^{\prime}+Z^{\prime}
\end{gathered}
$$

Реакции синтеза продукта

$$
\begin{gathered}
S \stackrel{k_{m}}{\longrightarrow} P_{1}, S^{\prime} \stackrel{k_{m}}{\longrightarrow} P_{1}^{\prime}, \\
S Z \stackrel{k_{m}}{\longrightarrow} P_{1} Z, S Z^{\prime} \stackrel{k_{m}}{\longrightarrow} P_{1} Z^{\prime} .
\end{gathered}
$$

Константа скорости ферментативного синтеза цепей $k_{\mathrm{M}}$ может быть оценена с помощью уравнения Михаэлиса-Ментен, $k_{\mathrm{M}}=V_{\max } / K_{\mathrm{M}}$, где $V_{\max }-$ максимальная скорость реакции, зависящая от активности фермента, $K_{\mathrm{M}}-$ константа Михаэлиса для ДНК-полимеразы $[10,11]$. Активность фермента - условная величина, неявно включающая в себя и его концентрацию.

В модели напрямую не учитываются нуклеотиды, что объясняется использованием большого избытка этих компонентов в реальных ПЦР системах и соответственно пренебрежимо малым изменением их концентрации в течение процесса. В том случае если в моделируемой системе различаются начальные концентрации комплементарных одноцепочечных фрагментов или какие-либо из констант ассоциации или диссоциации, то упростить данную систему не представляется возможным. В то же время если указанные параметры совпадают, то, следовательно, в каждый момент времени совпадают концентрации парных компонентов и продуктов синтеза двух комплементарных цепей. В этом в случае формально достаточно рассматривать синтез только на одной из комплементарных цепей ДНК, считая, что на второй цепи происходит все то же самое. С точки зрения модельной системы это означает возможность замены концентраций, содержащих штрихованные компоненты, на соответствующие нештрихованные: $C_{F^{\prime}}(t)$ на $C_{F}(t)$, $C_{F Z^{\prime}}(t)$ на $C_{F Z}(t)$ и т.д. В этом случае система кинетических уравнений может быть несколько упрощена и записана так:

$$
\begin{aligned}
\frac{d C_{F}}{d t} & =-k_{1} C_{F} C_{\mathrm{Pr}}+k_{-1} C_{S}-k_{1} C_{F} C_{Z}+k_{-1} C_{F Z} \\
& -k_{2} C_{F} C_{F}-k_{2} C_{S} C_{F}-k_{2} C_{F} C_{F Z}-k_{2} C_{F} C_{S Z},
\end{aligned}
$$

$$
\begin{aligned}
\frac{d C_{\mathrm{Pr}}}{d t} & =-k_{1} C_{F} C_{\mathrm{Pr}}+k_{-1} C_{S}-k_{1} C_{F Z} C_{\mathrm{Pr}}+k_{-1} C_{S Z} \\
& +k_{2} C_{S} C_{F}+k_{2} C_{F} C_{S Z}+k_{2} C_{S} C_{S}+k_{2} C_{S} C_{F Z} \\
& +2 k_{2} C_{S} C_{S Z}+k_{2} C_{S Z} C_{F Z}+k_{2} C_{S Z} C_{S Z}
\end{aligned}
$$$$
\frac{d C_{S}}{d t}=k_{1} C_{F} C_{\mathrm{Pr}}-k_{1} C_{S}-k_{1} C_{S} C_{Z}+k_{-1} C_{S Z}
$$$$
-k_{2} C_{S} C_{F}-k_{2} C_{S} C_{S}-k_{2} C_{S} C_{F Z}-k_{2} C_{S} C_{S Z}-k_{\mathrm{M}} C_{S},
$$$$
\frac{d C_{Z}}{d t}=-k_{1} C_{F} C_{Z}+k_{-1} C_{F Z}-k_{1} C_{S} C_{Z}+k_{-1} C_{S Z}
$$$$
+k_{2} C_{F} C_{F Z}+k_{2} C_{F} C_{S Z}+k_{2} C_{S} C_{F Z}+k_{2} C_{S} C_{S Z}
$$$$
+k_{2} C_{F Z} C_{F Z}+2 k_{2} C_{F Z} C_{S Z}+k_{2} C_{S Z} C_{S Z},
$$$$
\frac{d C_{F Z}}{d t}=k_{1} C_{F} C_{Z}+k_{-1} C_{F Z}-k_{1} C_{F Z} C_{\mathrm{Pr}}+k_{-1} C_{S Z}
$$$$
-k_{2} C_{F} C_{F Z}-k_{2} C_{S} C_{F Z}-k_{2} C_{F Z} C_{F Z}-k_{2} C_{F Z} C_{S Z},
$$$$
\frac{d C_{S Z}}{d t}=k_{1} C_{F Z} C_{\mathrm{Pr}}-k_{-1} C_{S Z}+k_{1} C_{S} C_{Z}-k_{-1} C_{S Z}-k_{2} C_{F} C_{S Z}
$$$$
-k_{2} C_{S} C_{S Z}-k_{2} C_{F Z} C_{S Z}-k_{2} C_{S Z} C_{S Z}-k_{\mathrm{M}} C_{S Z},
$$$$
\frac{d C_{P_{2}}}{d t}=k_{2} C_{F} C_{F}+2 k_{2} C_{S} C_{F}+2 k_{2} C_{F} C_{F Z}+2 k_{2} C_{F} C_{S Z}
$$$$
+k_{2} C_{S} C_{S}+2 k_{2} C_{S} C_{F Z}+2 k_{2} C_{S} C_{S Z}+k_{2} C_{F Z} C_{F Z}
$$$$
+2 k_{2} C_{F Z} C_{S Z}+k_{2} C_{S Z} C_{F} \text {, }
$$

$$
\frac{d C_{P 1}}{d t}=k_{\mathrm{M}} C_{S}, \quad \frac{d C_{P 1 Z}}{d t}=k_{\mathrm{M}} C_{S Z}
$$

где $C_{A}$ - концентрация компонента $A$.

Моделирование проводили с помощью программы, написанной на языке $\mathrm{C}++$. Система (1) решалась численно методом Рунге-Кутта четвертого порядка. Программа позволяла повторить модельные вычисления заданное количество раз, имитируя необходимое количество циклов. Для первого цикла ПЦР задавали начальную концентрацию цепей $C_{F}(0)$, праймеров $C_{\mathrm{Pr}}(0)$, зонда $C_{Z}(0)$ и длительность этапа элонгации $T$.

В конце цикла определялась концентрация синтезируемых ДНК без зонда $C_{P_{1}}(T)$ и с зондом $C_{P_{1} Z}(T)$, которая давала соответствующую точку на кривой ПЦР-РВ. Для следующего цикла увеличивалось количество исходной ДНК на величину $C_{P_{1}}(T)+C_{P_{1} Z}(T)$, на эту же величину уменьшалась начальная для следующего цикла концентрация праймера. Концентрация зонда уменьшалась на $C_{P_{1} Z}(T)$.

\section{Результаты}

Адекватность модели была проверена путем сопоставления экспериментальной кривой ПЦР и модельной кривой, полученной с теми же начальными данными. Для получения экспериментальной кривой был использован набор реагентов для анализа гена $F c$ фрагмента IgG иммуноглобулинового рецептора ІІІа человека ( FCGR3A, GenBank NG_009066.1) производства ЗАО „Синтол“ 


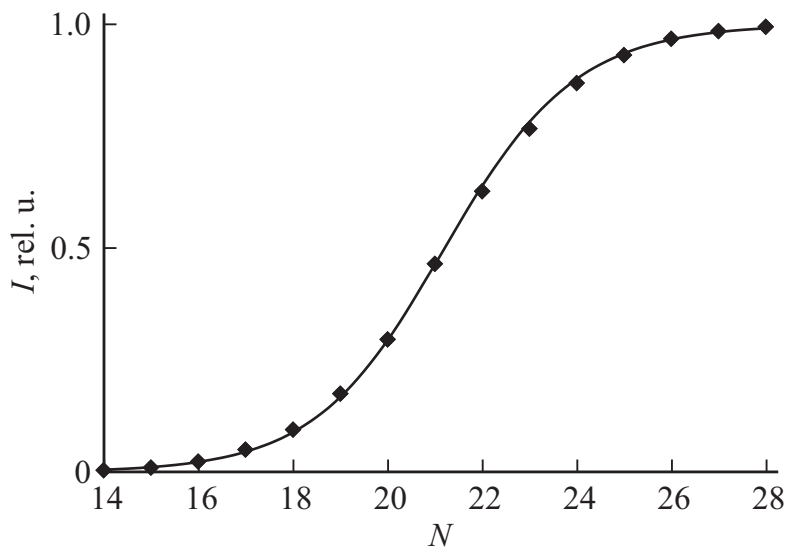

Рис. 1. Экспериментальная (ромбы) и теоретическая кривые ПЦР-РВ. Сплошная линия - теоретическая кривая отражает количество использованного зонда. Точка на экспериментальной кривой соответствует интегральной интенсивности флуоресцентного сигнала в реакционном объеме по завершению текущего цикла. $I$ - нормированная интенсивность флуоресцентного сигнала, $N$ - номер ПЦР цикла.

(Москва). Последовательности: прямой праймер Fc_up CACATATTTACAGAATGGCAAAGG; обратный праймер Fc_low CTGAAGACACATTTTTACTCCCAA; зонд Fc_Pr_up_ROX 5'-ROX-AGCGGCTCCTACTTCTG CAGGGG-RTQ2. Концентрация праймеров $\mathrm{C}_{\mathrm{Pr}}(0)=$ $=2 \cdot 10^{-7} \mathrm{M}$, зонда $C_{Z}(0)=1.2 \cdot 10^{-7} \mathrm{M}$, количество фермента на реакцию $-1 \mathrm{U}$.

Количество ДНК в модельном образце составляло $10^{7}$ копий на реакцию. Эксперимент был поставлен на амплификаторе CFX96 (Bio-Rad, CША). Параметры термоциклирования: предварительный нагрев до $95^{\circ} \mathrm{C}-$ $120 \mathrm{~s}$, далее 50 циклов: $62^{\circ} \mathrm{C}-30 \mathrm{~s}, 95^{\circ} \mathrm{C}-15 \mathrm{~s}$. Из практики известно, что данное время является достаточным для того, чтобы прошел полный синтез комплементарных цепей в процессе данной реакции. Детекцию результатов ПЦР проводили по каналу флуоресценции ROX.

В модельном эксперименте задавались те же исходные концентрации ДНК-мишени, зонда и праймера $C_{\operatorname{Pr}}(0), C_{Z}(0), C_{F}(0), \quad$ что и $\quad$ в эксперименте. Константы скорости реакций соответствовали проведению реакции в оптимальных физико-химических условиях $k_{11}=10^{6} \mathrm{M}^{-1} \mathrm{~s}^{-1}, \quad k_{21}=10^{6} \mathrm{M}^{-1} \mathrm{~s}^{-1}, \quad k_{2}=$ $=3 \cdot 10^{6} \mathrm{M}^{-1} \mathrm{~s}^{-1} \quad[10,11]$. Единице фермента соответствуют следующие значения параметров $V_{\max }=$ $=1 \cdot 10^{-8} \mathrm{Ms}^{-1}$ и $K_{\mathrm{M}}=2 \cdot 10^{-9} \mathrm{M}[10,11]$. Сравнение результатов численного и натурного экспериментов, представленное на рис. 1, свидетельствует об адекватности предложенной модели.

С помощью модели была исследована кинетика накопления всех копий исходной ДНК и их доли, внесшей вклад во флуоресцентный сигнал. Результаты анализа представлены на рис. 2. Приведенные данные свидетельствуют о том, что кумулятивные кривые накопления всего продукта реакции и его доли, увеличившей значение регистрируемого сигнала, могут заметно различаться. Разница между кривыми будет тем больше, чем больше разница соотношений исходной концентрации зонда и праймера. В случае если концентрация зонда соответствует концентрации праймера или превосходит ее, сопоставляемые кривые фактически не будут отличаться.
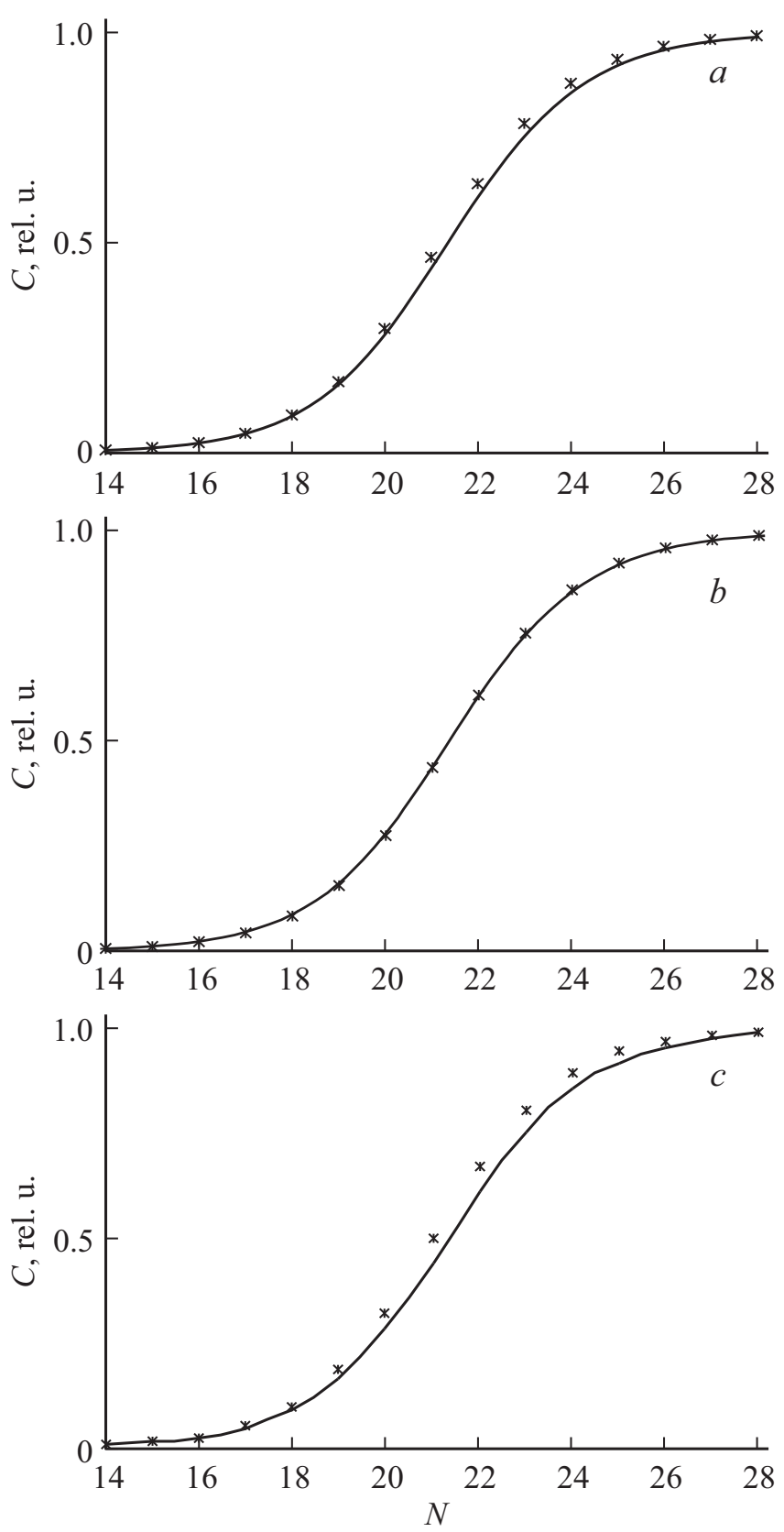

Рис. 2. Кумулятивные теоретические кривые накопления всех копий НК (сплошная линия) и их доли, внесший вклад в сигнал (звоздочки). $C-$ концентрация продукта, нормированная на максимальное значение, $N-$ номер ПЦР цикла. $a-$ концентрация зонда как в эксперименте $1.2 \cdot 10^{-7} \mathrm{M}$ (рекомендация производителя); $b-$ концентрация зонда в два раза выше рекомендуемой $-2.4 \cdot 10^{-7} \mathrm{M} ; c-$ концентрация зонда в два раза ниже рекомендуемой $-0.6 \cdot 10^{-7} \mathrm{M}$. 
На практике производители тест-систем обычно включают в реакционную смесь минимальное количество зонда, дающее достаточную амплитуду сигнала. Это связано с тем, что для дальнейшего анализа результатов включение большого количества зонда, например, равного или превышающего количество праймеров, не дает никаких преимушеств и лишь делает систему дороже. Таким образом, типичные экспериментальные кривые ПЦР-РВ нельзя напрямую сопоставлять с результатами количественного моделирования для продукта ПЦР-РВ копий исходной ДНК.

Работа выполнена в рамках государственного задания ФАНО России („Научно-методическое обеспечение разработок аналитических систем для нанобиотехнологии и диагностики социально значимых заболеваний и особо опасных инфекций на основе генетических, иммунных и физико-химических методов“", № 0074-2014-001).

\section{Список литературы}

[1] Gevertz J.L., Dunn S.M., Roth C.M. // Biotechnol. and Bioengineer. 2005. Vol. 92. N 3. P. 346-355.

[2] Lee J.Y., Lim H.W., Yoo S.I., Zhang B.T., Park T.H. // Biochem. Engineer. Journ. 2006. Vol. 29. N 1. P. 109-118.

[3] Smith M.V., Miller C.R., Kohn M., Walker N.J., Portier C.J. // BMC Bioinformatics. 2007. Vol. 8. N 1. P. 409.

[4] Cobbs G. // BMC Bioinformatics. 2012. Vol. 13. N 1. P. 203.

[5] Suzuki T., Fujikura K., Higashiyama T., Takata K. // Journ. Histochemistry \& Cytochemistry. 1997. Vol. 45. P. 49-53.

[6] Jones M.E., Mayne G.C., Wang T., Watson D.I., Hussey D.J. // BMC Bioinformatics. 2014. Vol. 15. P. 372.

[7] Lievens A., Van Aelst S., Van den Bulcke M., Goetghebeur E. // PLoS One. 2012. Vol. 11. e47112.

[8] Rutledge R.G., Stewart D. // PLoS One. 2010. Vol. 3. e9731.

[9] Liu W., Saint D.A. // Anal. Biochem. 2002. Vol. 302. P. 52-59.

[10] Сочивко Д.Г., Фёдоров А.А., Варламов Д.А. Курочкин В.Е., Петров Р.В. // ДАН. 2016. Т. 466. С. 109-113. [Sochivko D.G., Fedorov A.A., Varlamov D.A., Kurochkin V.E., Petrov R.V. // Doklady Biochemistry and Biophysics. 2016. Vol. 466. P. 13-16.]

[11] Сочивко Д.Г., Фёдоров А.А., Алексеев Я.И., Курочкин В.Е., Дубина М.В. // ДАН. 2017. Т. 472. С. 726-729. [Sochivko D.G., Fedorov A.A., Varlamov D.A., Kurochkin V.E., Petrov R.V. // Doklady Biochemistry and Biophysics. 2017. Vol. 472. P. 77-80.] 